\title{
Herramienta de evaluación de equipos biomédicos automatizados para laboratorios clínicos
}

\author{
Tatiana Chavarría Chavarría ${ }^{\psi}$ \\ Universidad CES. Medellin, Colombia
}

Recibido 28 de febrero de 2014. Aceptado 6 de marzo de 2017

\begin{abstract}
Resumen - Este artículo presenta una herramienta desarrollada como apoyo para los laboratorios clínicos en el proceso de evaluación y selección de tecnología biomédica, de forma que éste pueda ser automatizado en la fase analítica, facilitando la toma de decisiones basadas en la evidencia.

Para el desarrollo de la herramienta se estructuró un método que contó con la entrevista a expertos en gestión de tecnología biomédica de cuatro laboratorios clínicos del Departamento y se estableció una metodología de evaluación de tecnología en salud que facilita la toma de decisiones basadas en la evidencia, mediante un software que permite llevar a cabo el proceso de evaluación de tecnologías biomédicas automatizadas en laboratorios clínicos.

Como resultado se obtuvo un proceso estandarizado para evaluar tecnologías biomédicas automatizadas en laboratorios clínicos, realizando inicialmente un análisis de la necesidad real de tecnología que se tiene en la institución y posteriormente realizando los análisis técnico, clínico, económico y de proveedor a cada uno de los posibles equipos médicos automatizados que se pueden incorporar en las actividades del laboratorio y pueden solventar la necesidad de tecnología ya detectada.

Para llevar a cabo este proceso de manera sencilla y práctica, se diseñó un software que facilita la toma de decisiones en cuanto a la incorporación de equipos médicos al laboratorio clínico mediante la calificación de cada una de las características, especificaciones, atributos y funciones que brinda cada uno de los equipos que pueden solventar una necesidad especifica de tecnología biomédica.

El trabajo concluye con una estandarización y herramienta que presenta la ruta estratégica para adquirir tecnologías que satisfacen completamente las necesidades de un laboratorio clínico de manera que se mejoren y faciliten los procesos de evaluación tecnológica en éstos.
\end{abstract}

Palabras clave - Evaluación de tecnología biomédica, Laboratorio clínico, Software, Tecnología Biomédica Automatizada 


\section{Automated Health Technology Assessment Tool for Clinical Laboratories}

Abstract - The purpose of this paper is to describe a tool which will aid clinical laboratories standardize the process of Health Technology Assessment (HTA) and selection of automatic medical equipment at the analytical stage facilitating decision making.

The development of this tool was made by interviewing experts from four different clinical laboratories in our department. After this activity was complete we used a software to establish the based on evidence methodology to evaluate healthcare technology. We obtained a standardized process to evaluate automatic biomedical equipment in clinical laboratories by addressing the real technology need and then conducting a technical, clinical, economical and logistical analysis of each and every automatic medical equipment that could be used to solve that need.

In order to make HTA process that includes both the evaluation and selection appropriate, the software grade all medical equipment that could be used to solve the identified need by its characteristics, specifications, attributes and functions.

This work concludes with an alternative standardized method that could be used not only for the evaluation, selection and acquisition of appropriate automatic medical technology but also that improves and facilitates the evaluation process of these devices in clinical laboratories.

Keywords - Automated biomedical technology, Clinical laboratories, Health technology assessment, Software.

\section{FERRAMENTA DE AVALIAÇÃo DE TECNOLOGIA DE SAÚDE AUTOMATIZADA PARA LABORATÓRIOS CLÍNICOS}

Resumo - O objetivo deste trabalho é descrever uma ferramenta que auxiliará os laboratórios clínicos a padronizar o processo de Avaliação de Tecnologias em Saúde (HTA) e a seleção de equipamentos médicos automáticos no estágio analítico, facilitando a tomada de decisão.

O desenvolvimento desta ferramenta foi feito entrevistando especialistas de quatro laboratórios clínicos diferentes em nosso departamento. Depois que essa atividade foi concluída, usamos um software para estabelecer a metodologia baseada em evidências para avaliar a tecnologia de assistência médica. Obtivemos um processo padronizado para avaliar equipamentos biomédicos automáticos em laboratórios clínicos, abordando a real necessidade de tecnologia e, em seguida, realizando uma análise técnica, clínica, econômica e logística de cada um dos equipamentos médicos automáticos que poderiam ser usados para solucionar essa necessidade.

A fim de tornar o processo de ATS que inclua tanto a avaliação quanto a seleção apropriada, o software classifica todos os equipamentos médicos que poderiam ser usados para solucionar a necessidade identificada por suas características, especificações, atributos e funções.

Este trabalho conclui com um método padronizado alternativo que poderia ser usado não só para a avaliação, seleção e aquisição de tecnologia médica automática apropriada, mas também que melhora e facilita o processo de avaliação destes dispositivos em laboratórios clínicos.

Palavras-chave - Tecnologia biomédica automatizada, Laboratórios clínicos, Avaliação de tecnologia em saúde, Software.

\section{INTRODUCCIÓN}

$\mathrm{U}$ n laboratorio clínico es un área de la medicina básica indispensable en la actualidad debido a la labor que cumple en el diagnóstico clínico [1,2]. Los resultados de los exámenes de laboratorio ayudan a confirmar o descartar un diagnóstico, apoyan procesos de control de enfermedades y resultados de tratamientos, ayudan a detectar complicaciones, apoyan los estudios epidemiológicos y hacen parte de la investigación científica $[1,3]$.
Los procesos de un laboratorio clínico se dividen en tres etapas:

- Fase pre-analítica: abarca todas las acciones desde que el médico solicita el examen, las indicaciones que debe seguir el paciente para la toma de la muestra, la selección de los materiales, la toma de la muestra en el laboratorio, su transporte, almacenamiento hasta el momento del análisis y procesos de preparación de la muestra como centrifugación $[4,5]$. 
- Fase analítica: abarca todas las acciones para realizar el análisis, como son la selección de métodos y equipos (incluyendo procesos de calibración y mantenimiento asociados), sistema de control de calidad para la detección de errores analíticos, acciones correctivas, control de precisión y exactitud y el desarrollo de las técnicas $[4,5]$.

- Fase post-analítica: incluye procesos relacionados con la verificación de resultados, intervalos o rangos de referencia de la población, la puntualidad en la entrega de resultados, el informe del laboratorio y la confidencialidad de la información de los resultados. $[4,5]$.

La automatización busca transferir a una tecnología, tareas de cada fase de producción que son realizadas por humanos $[6,7]$. Para los laboratorios clínicos se tienen dos modelos de automatización, la primera es un laboratorio totalmente automatizado, es decir que se automatiza desde la fase pre analítica hasta la fase post analítica; este modelo es de poca aplicación ya que implica altos costos y una alta inversión inicial [8].

La otra modalidad es la llamada laboratorio automatizado modular, en la cual se crean áreas que, aunque están físicamente separadas, se agrupan por módulos de automatización denominados workcells dependiendo del tipo de muestra a analizar. Esta modalidad no requiere alta inversión pero trae desventajas como la necesidad de transportar muestras manualmente debido a la separación de los sistemas [8].

La evaluación de la tecnología en salud (ETES) está definida como la manera de determinar las consecuencias técnicas, clínicas, sociales, económicas, éticas y legales que produce en el corto y largo plazo la incorporación, desarrollo, difusión y uso de una tecnología sanitaria $[9,10]$. La evaluación de tecnologías en salud se encarga de realimentar procesos de gestión de tecnología tales como la planeación y el control con el fin de tomar decisiones que mejoren la eficiencia y la calidad en la prestación de los servicios [11].

La evaluación tiene como finalidad determinar el valor de una tecnología teniendo en cuenta aspectos como su seguridad, eficiencia, efectividad, manejo del riesgo y beneficio, componentes financieros y competitividad [12-14]. No es únicamente una evaluación de la tecnología, es también una herramienta para la toma de decisiones de planeación y administración en una institución de salud [15].

La adquisición de equipos biomédicos es una etapa del procedimiento de evaluación de la tecnología en salud donde se realizan planes y programas de compras de los equipos médicos según necesidades, prioridades y disponibilidad de recursos $[13,15]$. Los criterios que se deben tener en cuenta en la evaluación de una tecnología biomédica para un laboratorio clínico son el clínico, el económico y el técnico $[16,17]$.

El clínico se basa en criterios subjetivos, como lo son la utilidad, la confiabilidad y los factores asociados a su uso [17-19]. El criterio económico evalúa aspectos relacionados con costos implícitos en el uso del equipo, como el análisis de reposición y los costos de mantenimiento y operación del equipo $[16,20,21]$. Por último el criterio de evaluación técnico se enfoca en los aspectos relacionados con el ámbito funcional equipo, teniendo en cuenta la edad del equipo, el tiempo e intensidad de uso, el manual de usuario y el mantenimiento y suministros que el equipo necesitará en su vida útil [16, 21].

Este trabajo presenta una metodología que facilita el proceso de evaluación de tecnologías biomédicas automatizadas para laboratorios clínicos; se propone inicialmente una evaluación a la necesidad de tecnología automatizada que tienen los laboratorios clínicos y a todas las posibles soluciones que se tienen para solventar dicha necesidad, igualmente se presenta un método que permite seleccionar la tecnología biomédica más apropiada para el laboratorio clínico siguiendo el análisis de parámetros técnicos, económicos, clínicos y de proveedor.

\section{Materiales y Métodos}

Para la selección de los laboratorios clínicos se ubicaron aquellos que se encuentran en la ciudad de Medellín y se priorizaron seis (6) de acuerdo con las siguientes características: mayor volumen y flujo de pacientes, cantidad y diversidad de equipos (mayor cantidad y tipos de pruebas que pueden realizarse) y mayor número de convenios o contratos con EPS.

Posterior a este filtro, se inició el contacto directo con el personal a cargo del tema de gestión de tecnología biomédica automatizada dentro de estos laboratorios clínicos. Estos profesionales hacen parte del equipo que realiza la adquisición de nueva tecnología biomédica, por lo tanto conocen el proceso de selección, evaluación y adquisición.

Dentro de estos laboratorios clínicos seleccionados, se encuentran cinco (5) de carácter público y uno (1) privado que depende y funciona dentro de una clínica.

Se les pidió a los expertos responder una serie de preguntas relacionadas con la secuencia seguida por el laboratorio clínico en los procesos de adquisición de nuevas tecnologías biomédicas automatizadas. Después de identificados los requerimientos que se tienen dentro de los laboratorios clínicos y las expectativas de los usuarios de las tecnologías biomédicas, se procedió a seleccionar 
los componentes de la evaluación de tecnología para adquisición de equipos analizadores automatizados para laboratorios clínicos.

Con esta información se realizó un diagrama de flujo que resume la secuencia del proceso de selección de equipos médicos.

Finalmente, se realizó un software utilizando la herramienta Visual Basic para aplicaciones versión 6.0 integrada a Microsoft Excel.

El editor de Visual Basic integrado a Excel contiene las herramientas de programación que permitieron escribir código usando lenguaje de programación Visual Basic y crear una interfaz gráfica amigable que facilita el uso del software.

El software contiene una serie de formularios que permiten al laboratorio clínico realizar la evaluación de manera organizada, luego la información suministrada es almacenada en hojas de Excel a manera de base de datos, finalmente, el software realiza una serie de cálculos y presenta los resultados de la evaluación en tablas dinámicas.

\section{ResUltados}

Se encontró que los laboratorios clínicos de Medellín prefieren adquirir su tecnología biomédica mediante contratos de comodato o leasing ya que en esta forma de financiación encuentran la posibilidad de hacer renovación tecnología con mayor frecuencia, además el proveedor es el responsable del funcionamiento del equipo y el suministro de reactivos.

Los laboratorios clínicos necesitan que los equipos biomédicos que se incorporan en sus procesos suplan la carga de trabajo de la institución, es decir que la tecnología pueda procesar la cantidad de muestras que son solicitadas diariamente. Prefieren aquellos proveedores que suministran sus equipos biomédicos con equipos de cómputo, sistemas de alimentación eléctrica ininterrumpida, reactivos, controles de calidad y demás consumibles. Para los usuarios de los equipos biomédicos es importante que el control de calidad de cada prueba sea estable y que los equipos sean novedosos y de última tecnología.

Teniendo en cuenta los anteriores requerimientos detectados en las entrevistas con los expertos de los laboratorios clínicos, se propone una metodología de evaluación de tecnología para adquisición de equipos médicos por parte de estos últimos, basada en cinco componentes: evaluación de las necesidades, evaluación técnica, evaluación económica, evaluación clínica y evaluación de proveedor (Fig. 1).
El proceso de evaluación comienza cuando el usuario de una tecnología detecta una necesidad en determinada area (Fig. 1). Al detectarse la necesidad, el laboratorio debe conformar un comité de evaluación de tecnología que se encargará inicialmente de evaluar la necesidad y clasificarla para darle la solución adecuada. Se sugiere que este comité este conformado por personal del área médica (director o jefe médico, jefe del laboratorio clínico o jefe de procesos, jefe del sistema de control de calidad del laboratorio, profesionales de laboratorio clínico y auxiliares de laboratorio que están en el proceso al que entrará la tecnología), área administrativa y financiera, área de infraestructura o ambiente físico y área de mantenimiento e ingeniería.

Inicialmente, el comité de evaluación de tecnología deberá realizar el proceso de evaluación de la necesidad; primero se evalúan condiciones de salud pública de la población a atender con la nueva tecnología: se incluyen preguntas sobre la prevalencia y la prioridad de las enfermedades cuyo diagnóstico se facilitará con la incorporación de dicha tecnología en los procesos analíticos del laboratorio clínico.

Otro parámetro a tener en cuenta en la evaluación de la necesidad es la disponibilidad de servicios prestados por el laboratorio, esto con el fin de estudiar las pruebas que pueden hacerse en cada sección del laboratorio, el número de centrales de procesamiento y de muestras remitidas a otros laboratorios y las causas por las que se dan estas remisiones. Lo anterior permite realizar un análisis de la prestación del servicio, la Fig. 2 muestra los formularios que presenta el software y que deben ser diligenciados por el comité de evaluación de tecnología.

También se recomienda evaluar la disponibilidad de equipos biomédicos analizadores en las centrales de procesamiento, además de las condiciones de infraestructura de la institución, para analizar parámetros relacionados con los aires acondicionados, edificios, conexiones e instalaciones eléctricas, sistema de abastecimiento de agua, suministro de energía en caso de emergencia y cantidad de equipos biomédicos por sección, como se muestra en la Fig. 3.

No se puede dejar de lado el recurso humano disponible por el laboratorio, ya que estudiando dicho parámetro se pueden detectar habilidades del personal para procesar con la nueva tecnología, o por el contrario se puede determinar la necesidad de contratar nuevo personal para garantizar que la nueva tecnología va a ser operada de manera eficaz, es por esto que el software también incorpora una sección para el estudio del recurso humano disponible.

La adquisición o no de una tecnología biomédica para la institución depende en parte de la situación económica 
que se tenga y de la que se espera, muchos de las instituciones de salud de nuestra ciudad disponen de recursos limitados para adquirir o renovar su tecnología por lo que el factor económico es uno de los más importantes a la hora de adquirir una tecnología nueva, sin embargo una adquisición no será valiosa si no se reconocen los beneficios (no solo tangibles) que obtendrá la institución por reemplazo de tecnologías.

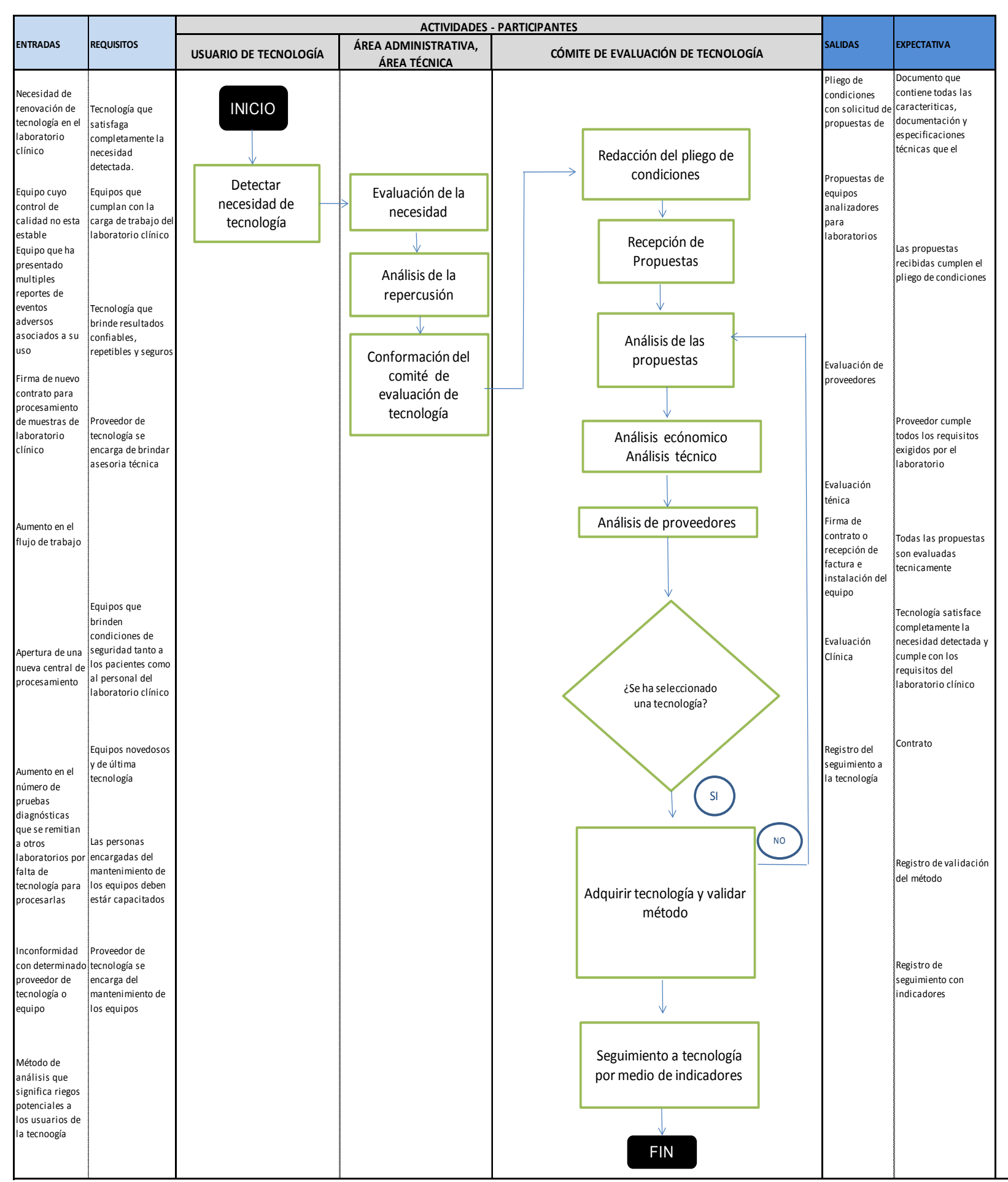

Fig. 1. Metodología de evaluación de tecnología para adquisición de equipos biomédicos en laboratorios clínico 


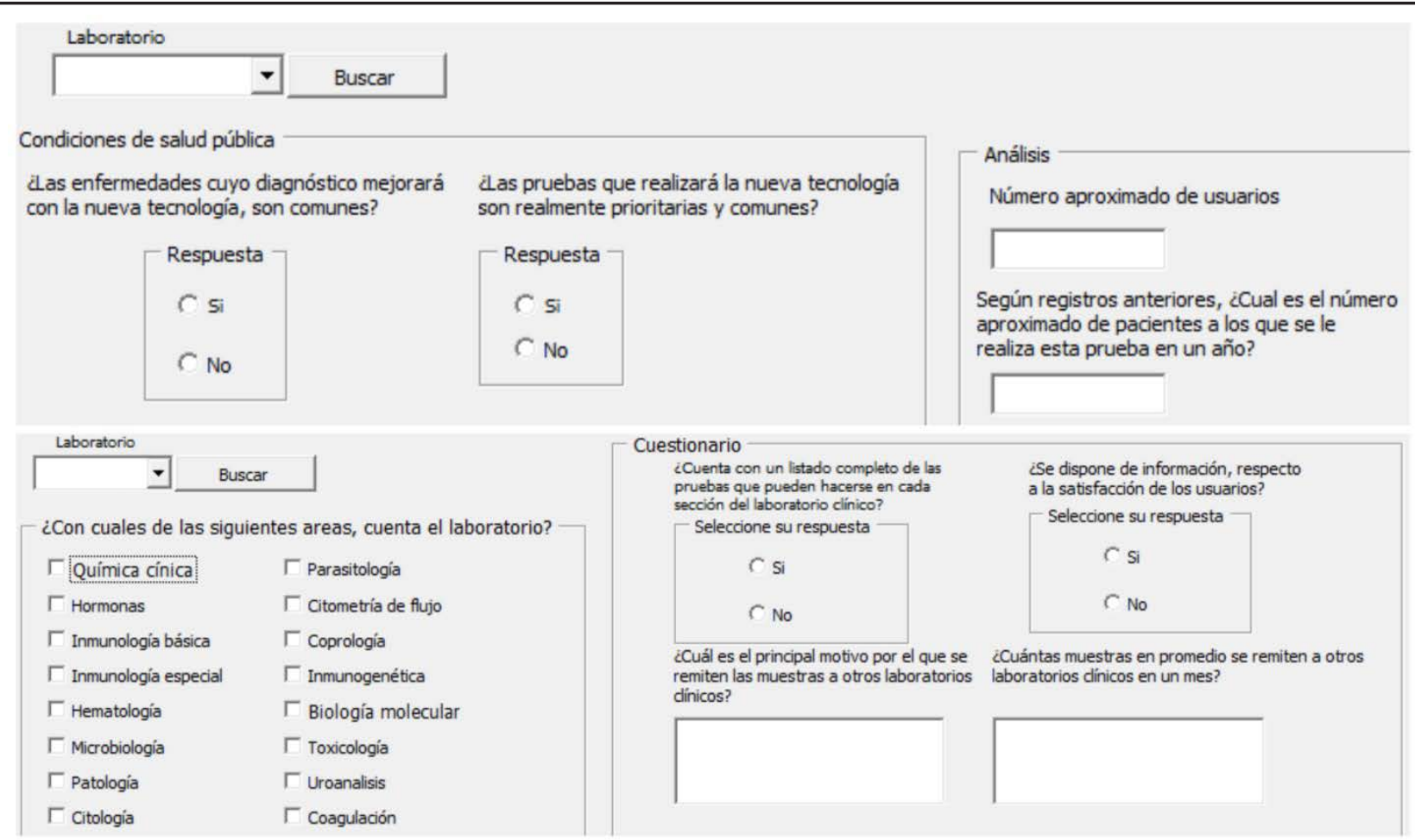

Fig. 2. Formularios para Evaluación de las condiciones de Salud Pública y la disponibilidad de servicios en el laboratorio clínico.

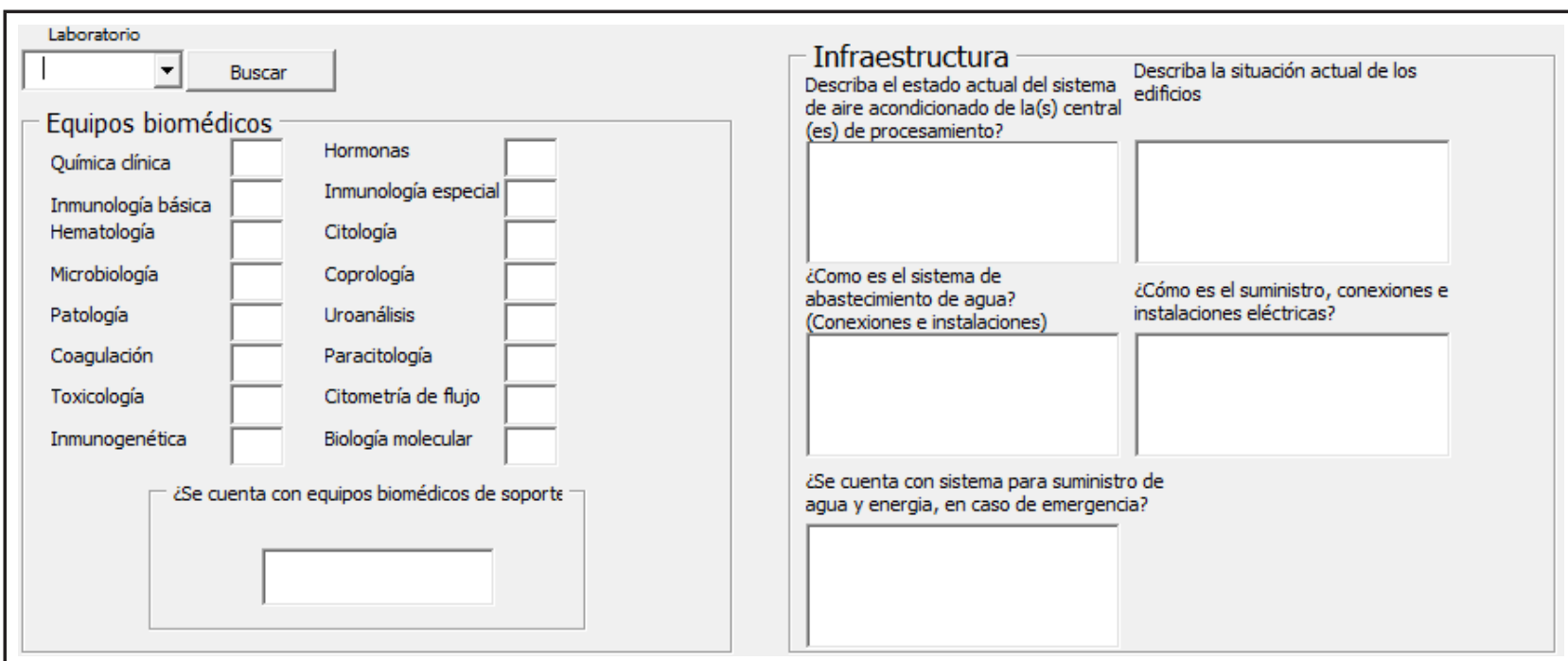

Fig. 3. Formulario para la evaluación de la disponibilidad de equipos biomédicos y las condiciones de infraestructura.

Finalmente, se procede con la evaluación de la repercusión de la nueva tecnología biomédica en los procesos del laboratorio clínico y la toma de decisiones. En ella se incluyen preguntas relacionadas con beneficios del ingreso de la nueva tecnología en cuanto a disminución del tiempo de procesamiento, facilidad de procesamiento, competitividad y posicionamiento del laboratorio clínico, mejora en la calidad de la prestación de los servicios, disminución de riesgos y eventos adversos, disminución de gastos y optimización de costos e impactos positivos en el cuidado al medio ambiente (Fig. 4). Estos aspectos son calificados usando la escala que se presenta en la Fig. 5, que se realiza para cada una de las opciones que se tiene para solventar la necesidad detectada. 


\begin{tabular}{|c|c|c|c|}
\hline \multirow{2}{*}{$\begin{array}{l}\text { Variable de evaluación de } \\
\text { repercusión }\end{array}$} & \multicolumn{3}{|c|}{ Calificación } \\
\hline & Opción 1 & Opción 2 & Opción 3 \\
\hline \multicolumn{4}{|l|}{$\begin{array}{l}\text { Beneficios de la opción, en disminución } \\
\text { de tiempo de procesamiento. }\end{array}$} \\
\hline \multicolumn{4}{|l|}{$\begin{array}{l}\text { Beneficios de la opcion en facilidad de } \\
\text { procesamiento de muestras. }\end{array}$} \\
\hline \multicolumn{4}{|l|}{$\begin{array}{l}\text { Beneficios de la opción en } \\
\text { competitividad y posicionamiento del } \\
\text { laboratorio. }\end{array}$} \\
\hline \multicolumn{4}{|l|}{$\begin{array}{l}\text { Beneficios de la opción en renovación } \\
\text { de tecnología y mejora en la calidad del } \\
\text { servicio. }\end{array}$} \\
\hline \multicolumn{4}{|l|}{$\begin{array}{l}\text { Beneficios de la opción en disminución } \\
\text { de riesgos, o posibles eventos } \\
\text { adversos. }\end{array}$} \\
\hline \multicolumn{4}{|l|}{$\begin{array}{l}\text { Beneficios de la opción en disminución } \\
\text { de gastos y optimización de costos. }\end{array}$} \\
\hline \multicolumn{4}{|l|}{ Opción cumple requisitos legales } \\
\hline \multicolumn{4}{|l|}{$\begin{array}{l}\text { Impáctos positivos de la opción, en el } \\
\text { cuidado del medio ambiente. }\end{array}$} \\
\hline & Total & Total & Total \\
\hline
\end{tabular}

Fig. 4. Evaluación de la Repercusión de cada solución.

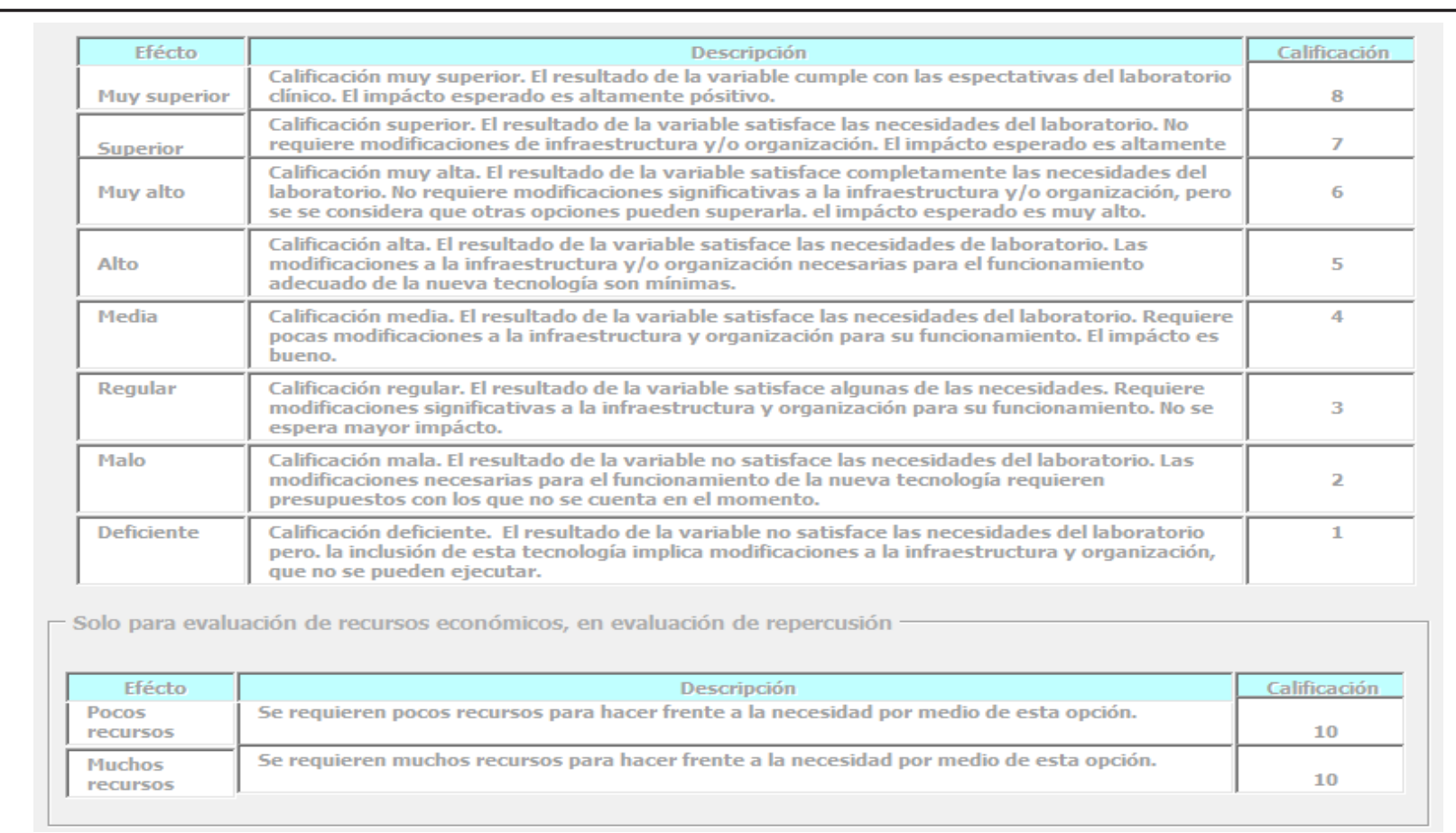

Fig. 5. Escala de calificación

Igualmente, en la evaluación de repercusión se incluye un componente de análisis de recursos (humanos, físicos, económicos), en la que se incluyen los requerimientos para hacer frente a la necesidad por medio de cada opción (Fig. 6). La calificación se realiza siguiendo la escala presentada en la Fig. 2.
El software realiza el cálculo tanto de la evaluación de la repercusión como de la evaluación de recursos. Con esta información el laboratorio deberá determinar si la adquisición de una nueva tecnología es o no la solución apropiada para la necesidad encontrada. Se recomienda que el laboratorio se incline por la opción que requiere 
pocos recursos para realizar el cambio y cuya repercusión sea mayor como se muestra en la Fig. 7.

$\mathrm{Si}$ el laboratorio ha decidido adquirir una nueva tecnología después del análisis de la necesidad, comenzará entonces el proceso de evaluación de tecnología aplicada a cada una de las opciones recibidas de los diferentes proveedores participantes.

La evaluación para adquisición de equipo biomédico en el laboratorio clínico tiene tres evaluaciones: técnica, económica y de proveedor; igualmente, se propone realizar una evaluación clínica del método de análisis de la nueva tecnología.

\section{Evaluación técnica}

Permite calificar las especificaciones de las propuestas recibidas, se califican accesorios, características físicas y requerimientos de infraestructura (el formulario del software permite realizar este proceso, ver Fig. 8). Cada una de las características es evaluada usando la escala presentada en la Fig. 2.

El comité hace la calificación y finalmente calcula el total para cada equipo o propuesta y guarda la información.

\section{Evaluación económica}

Se sugiere un análisis de costo - beneficio para cada una de las propuestas mediante la calificación de una serie de parámetros que permiten comparar el impacto percibido por la institución con relación a la inversión realizada. Igualmente se incorpora formulario que facilita el análisis del costo del contrato para cada uno de los proveedores.

\begin{tabular}{|c|c|c|c|}
\hline \multirow{2}{*}{$\begin{array}{l}\text { Variable de evaluación de } \\
\text { repercusión }\end{array}$} & \multicolumn{3}{|c|}{ Calificación } \\
\hline & 0pción 1| & 0pción 2 & 0pción 3 \\
\hline $\begin{array}{l}\text { Recursos necesarios para realizar el } \\
\text { cámbio. Por medio de esta opción son } \\
\text { altos. }\end{array}$ & & & \\
\hline $\begin{array}{l}\text { Recursos necesarios para realizar el } \\
\text { cámbio. Por medio de esta opción son } \\
\text { bajos. }\end{array}$ & & & \\
\hline
\end{tabular}

Fig. 6. Evaluación de los recursos necesarios en cada solución.

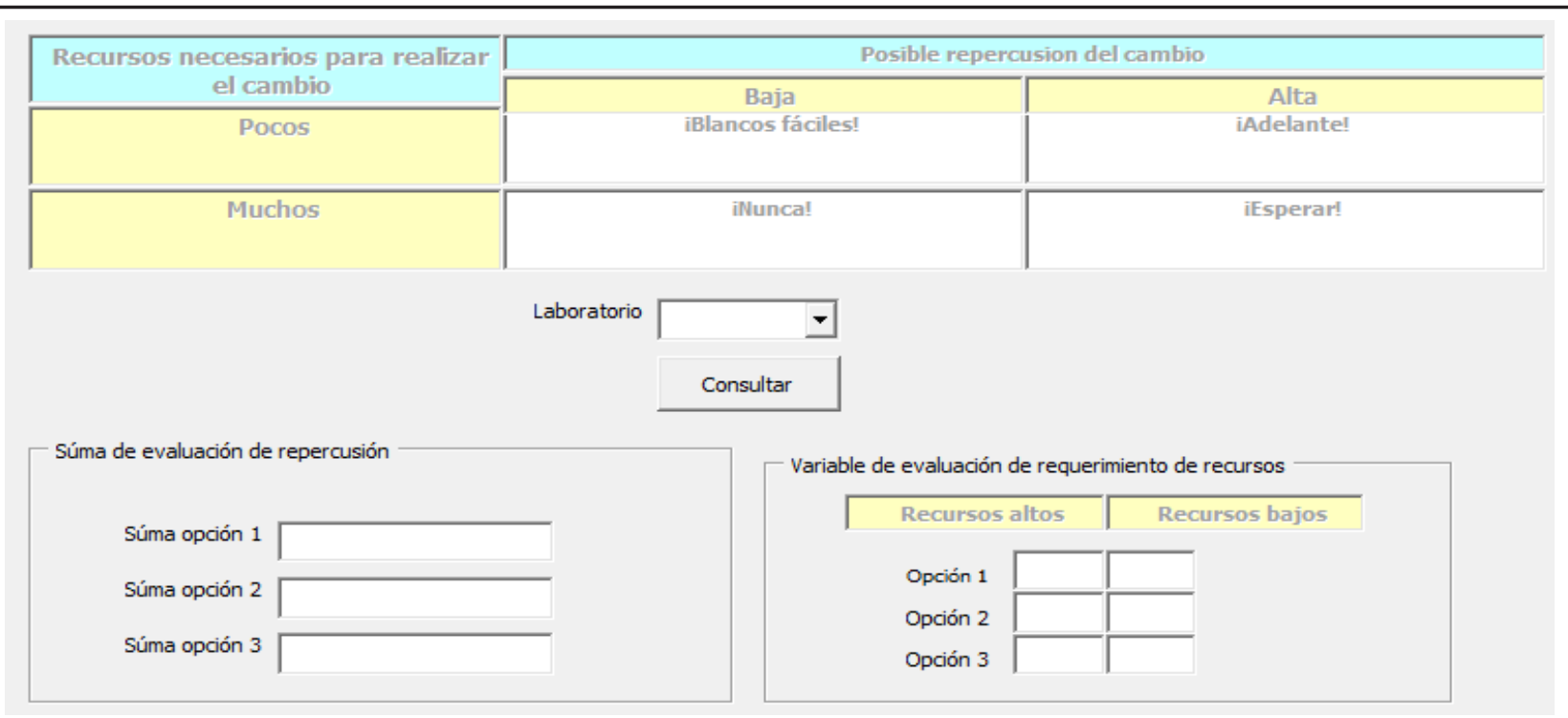

Fig. 7. Criterios para la toma de la decisión. 


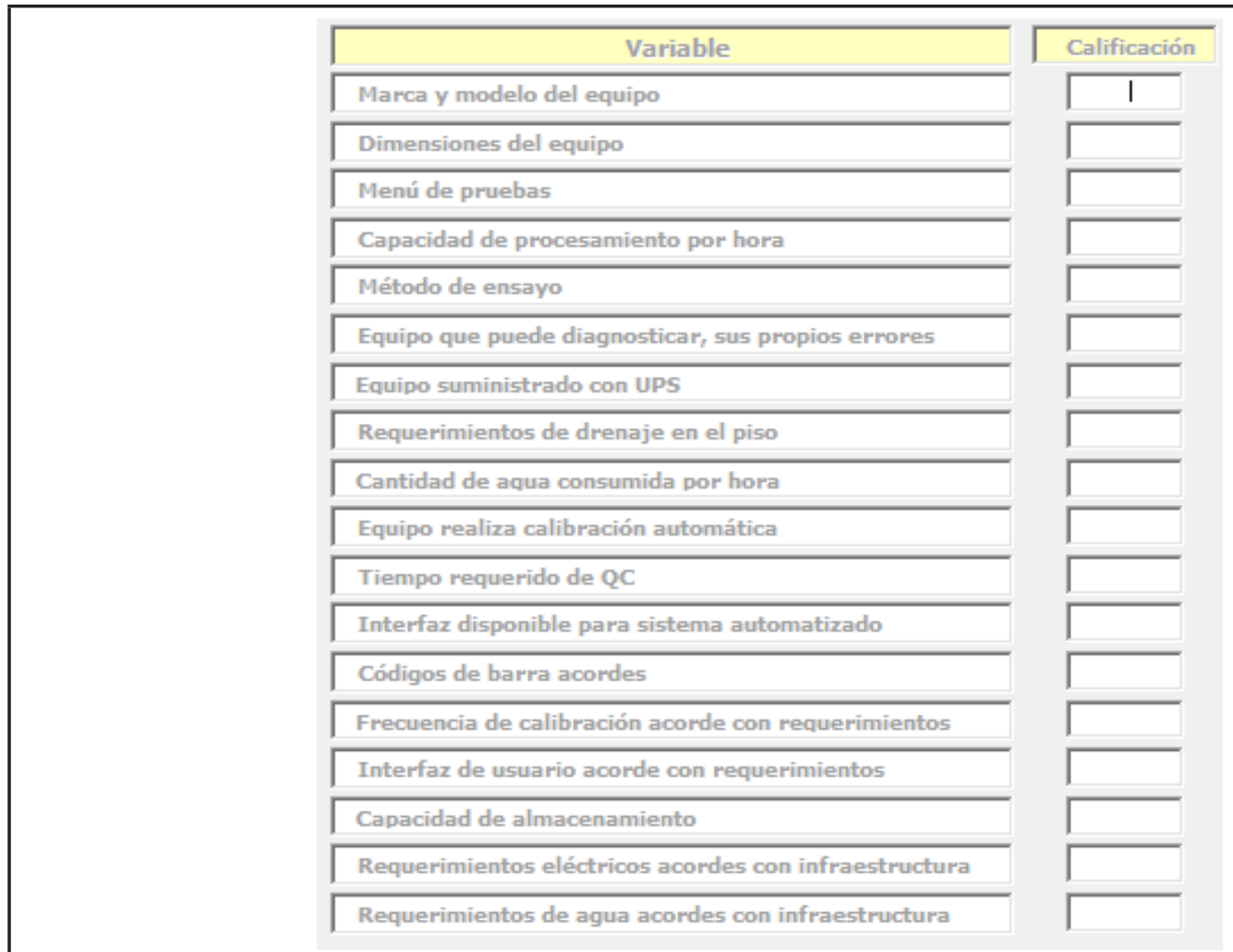

Fig. 8. Evaluación Técnica

\section{Evaluación del proveedor}

El software incluye un formulario que permite calificar ciertas características del proveedor que los laboratorios clínicos consideran importantes antes de realizar un contrato, esto permite seleccionar proveedores con suficiente experiencia y personal capacitado para realizar todas las tareas de soporte, asesoría y gestión de la tecnología.

\section{Resultado de la evaluación de tecnología}

Finalmente, el software presenta los resultados de las evaluaciones mediante una tabla dinámica de Excel donde se observan los resultados de las calificaciones de cada uno de los componentes de la evaluación (técnica, económica y de proveedor) para cada una de las propuestas presentadas, en la Tabla 1 se presentan las características generales del Software desarrollado.

\section{Evaluación clínica}

La selección de un equipo biomédico para un laboratorio clínico depende del método de análisis el cual debe satisfacer las expectativas de la institución; para garantizar esto se propone incluir una evaluación clínica que tenga en cuenta el análisis de la sensibilidad analítica y la especificidad, términos que están ligados a la calibración, el intervalo de referencia (valores normales), la precisión, la exactitud y las sustancias que causan interferencia en el análisis; para esto se recomienda solicitar el equipo en demostración inicialmente e intercambiar opiniones para conocer las características de los diagnósticos de una enfermedad en particular.

Los parámetros que se pueden considerar mediante una evaluación clínica son: la precisión, exactitud, linealidad, límite de detección, especificidad y estabilidad de los reactivos.

Los resultados que se obtienen de la validación de un método se pueden usar para juzgar la calidad, fiabilidad y consistencia de los resultados analíticos, además de que es una parte integral de las buenas prácticas en el análisis de laboratorio clínico.

Este proceso es útil para confirmar que el método tiene capacidades de rendimiento adecuadas, permite verificar que el equipo está dentro de las especificaciones, funciona correctamente y esta adecuadamente calibrado.

La evaluación clínica se recomienda pero es específica para cada laboratorio ya que este es quien selecciona que controles, calibradores y laboratorios de referencia utilizar. 
Tabla 1. Características Generales del Software de Evaluación de Tecnologías para Laboratorios clínicos.

\section{NOMBRE}

SEQUILAB (Sistema de Evaluación de Equipos de Laboratorio)
CARACTERISTICAS DEL DESARROLLO

Desarrollado en Visual Basic para aplicaciones incorporado en Microsoft Excel.

\begin{tabular}{lll}
\hline & CARACTERISTICAS TÉCNICAS \\
\hline Requerimientos de uso & - & Tener acceso a Microsoft Excel. \\
& - & Tener Activada la opción "PROGRAMADOR" de Microsoft Excel. \\
\hline & FUNCIONALIDADES
\end{tabular}

- El software tiene en cada uno de los formularios cinco botones con las siguientes funcionalidades: crear un Nuevo registro, Guardar, Eliminar y/o Modificar información suministrada.

- El software presenta inicialmente un formulario donde el Laboratorio clínico deberá registrarse suministrando la siguiente información: Nit, razón social, teléfono y dirección.

Botones principales

- En cada formulario del proceso de evaluación, el usuario deberá buscar el Nit de su institución en la lista desplegable llamada "Laboratorio".

- Cada vez que se ingrese información al software y antes de pasar a otro formulario, el usuario deberá Guardar, de lo contrario los datos se perderán.

- En los formularios finales, el usuario deberá dar click en el botón Calcular para que el software arroje el resultado cuantitativo de cada evaluación.

El software contiene los siguientes formularios para realizar el proceso de evaluación de la necesidad de tecnología biomédica: Identificación del Laboratorio, Condiciones de Salud Pública, Disponibilidad de Servicios, Disponibilidad de equipos y estado de infraestructura, Recursos humanos, Repercusión y evaluación de la repercusión, esta última a la vez contiene los siguientes formularios: Variables de evaluación de la repercusión, Evaluación del requerimiento de recursos y Toma de decisión. Después, el software presenta los siguientes formularios para la evaluación de tecnologías biomédicas: Evaluación de propuestas, Evaluación Económica, Evaluación técnica y Evaluación de Proveedor.

Finalmente el Software da enlace a una carpeta en la nube que contiene las especificaciones técnicas de los principales equipos de laboratorio clínico presentes en el mercado Colombiano.

\section{Discusión}

Los resultados obtenidos en este trabajo se pueden extender a otros contextos, esto quiere decir que con algunos cambios en el diseño de la metodología planteada se puede lograr que esta propuesta sea empleada por hospitales, clínicas y en general otras instituciones de salud diferentes a los laboratorios clínicos.

Si se comparan los resultados obtenidos en este trabajo con el documento metodológico de evaluación de tecnologías para la salud del Centro Nacional de Excelencia Tecnológica en Salud (CENETEC) de México [22], se pueden encontrar similitudes en el proceso ya que en ambos considera que la evaluación clínica, la evaluación económica y la evaluación social, ética y organizacional se deben tener en cuenta en el momento de seleccionar y adquirir una nueva tecnología biomédica; para este trabajo los aspectos sociales, de ética y organizacionales no se llamaron de esa manera pero si se consideraron en la etapa de evaluación de la necesidades reales de tecnologías biomédicas.

La evaluación económica es considerada como uno de los aspectos más importantes cuando se va a adquirir una nueva tecnología biomédica, esto principalmente por la escases de recursos de algunas instituciones de salud, así lo dice el Departamento de Sanidad del Gobierno Vaco en su Guía de Evaluación Económica en el Sector Sanitario [23], nuestro proceso para adquisición de equipos biomédicos para laboratorios clínicos se propuso analizar integralmente la disponibilidad de recursos de la institución, y para nuestro caso no solo recursos económicos sino también recursos humanos y de infraestructura física como lo propone el documento técnica de la OMS para la evaluación de las necesidades de dispositivos médicos [24].

La evaluación clínica siempre se debe tener en cuenta en el proceso de adquisición de tecnologías biomédicas, sin embargo no en todas las metodologías propuestas a 
través de los años esta evaluación ha tomado este nombre ya que en muchos se ha denominado como la evaluación enfocada a la seguridad del paciente.

Para este trabajo se propone adicionalmente evaluar a los proveedores de la tecnología biomédica a adquirir, esta evaluación no se encontró en ninguna de las referencias bibliográficas consultadas pero los expertos entrevistados en la ciudad lo consideraron de gran importancia.

El Centro Danés de Evaluación de Tecnologías en salid (DACHETA) publicó en 2007 el manual de Evaluación de Tecnologías más completo, robusto y usado hoy día [25], este manual considera la evaluación como un proceso integral entre las categorías de ética, tecnología, paciente, organización y economía; el manual considera la ética como la relación entre en médico y el paciente teniendo en cuenta el respeto por la autonomía, la no maleficencia, la beneficencia y la justicia, sin embargo en nuestro estudio la ética tenida en cuenta de esta manera no fue considerada ya que no hay una relación directa entre el paciente, el médico y el equipo biomédico automatizado; sin embargo para futuros trabajos de este tipo, se sugiere incorporar la ética como la relación entre los datos arrojados por la tecnología biomédica, el médico y el paciente.

La evaluación de las necesidades busca comparar la situación actual y la deseada al adquirir una tecnología nueva, para este proceso se siguió la metodología planteada por la OMS en su documento "Evaluación de las necesidades de dispositivos médicos", sin embargo, se propuso el proceso siguiendo los lineamientos propuestos por los laboratorios clínicos consultados y las características de estos [24].

Otro trabajo realizado en Medellín en el año 2008 [26], considera la importancia de la conformación de comités de evaluación de tecnologías para asegurar que la solución a las necesidades planteadas sea la más óptima, además de contar con suficientes capacidades y criterios para tomar la decisión, en nuestro trabajo igualmente se propuso conformar un comité de evaluación de tecnología que permitiera la interacción entre las diferentes áreas del laboratorio clínico que tienen relación con la adquisición de nuevas tecnologías.

La implementación de la metodología planteada en este trabajo puede llegar a significar un gran avance hacia la mejora en la toma de decisiones en cuanto a la selección de tecnología biomédica que se acople a las verdaderas necesidades de una institución de salud.

\section{REFERENCIAS}

[1]. Alfonso de León, A. G. Utilización inadecuada de los avances científicos técnicos del laboratorio clínico y del método clínico. Revista Médica Electrónica. 2013. 35(4).
[2]. Pérez Valero, V. El Laboratorio Clínico en el Sistema Asistencial. SEMERGEN.2011. 11-112.

[3]. Jiménez, I. La importancia de los exámenes de laboratorio clínico de rutina o de prevención. Diario Moderno y Profesional Opinión. Consultado el 26 de marzo de 2019 en: https://www.diariopinion. $\mathrm{com} / \mathrm{salud} /$ verArticulo.php?id=829047

[4]. Pabón Martínez, H., \& Londoño Núñez, P. A. Plan de Mejoramiento para disminuir los errores en la fase pre analítica en los análisis de laboratorio en la Clínica Regional de Occidente de la Policía Nacional seccional Sanidad Valle de la ciudad de Santiago de Cali. Cali: Universidad Cooperativa de Colombia. 2018.

[5]. Coronado Herrera, Y., arbalo Rivero, M., Abreu Correa, M., Garbosa Savón, K., Fariñas, O., \& García Herrera, A. Importancia de la fase preanalítica en el laboratorio clínico de la Atención Primaria en Salud. Revista de Medicina Isla de la Juventud. 2014. 15(1), 1-19.

[6]. Escuela Universitaria de Ingeniería Técnica Industrial de Eibar. Automatización. Universidad del País Vasco. Consultado el 27 de marzo de 2019 en: http://www.sc.ehu.es/sbweb/webcentro/automatica/WebCQMH1/PAGINA\%20PRINCIPAL/Automatizacion/ Automatizacion.htm

[7]. Escalona M, Miranda A, Acosta A. Automatización en el laboratorio clínico. Revista Chilena de Tecnología Médica. 2009. 29 (2): 1521-1526.

[8]. Bedini Chesa JL, Esteve Poblador S, García Beltrán L, Gasalla Herraiz JM, Macías Blanco C, Martinez Casademont M, Moreno Cebeira JM, Martínez Vásquez V, Prieto García B, Serrano Olmedo G, Torres Nicolau J. Criterios para la Selección de un Modelo de Automatización del Laboratorio. Documento A. Fase 2. Versión 2. Sociedad Española de Bioquímica Clínica y Patología Molecular. 2009.

[9]. Escobar, N. Biblioteca Virtual en Salud CUBA. Consultado el 27 de marzo de 2019 en: http://www.bvs.sld.cu/revistas/san/ vol3_1_99/san01199.pdf

[10]. Secretaría de Salud de México. Evaluación de Tecnologías para la Salud. México D.F; 2010.

[11]. Molina Velasquez T. Ingeniería Clínica para no Ingenieros: Adquisición de Equipos Médicos. Revista Ingeniería Biomédica. 2007. (2): 40-47.

[12]. AHFMR. A Guide to Health Technology Assessment in the Palliser Health Region. Canadá. Junio 2006.

[13]. Salazar Flórez, K. J., Botero Botero, S., \& Jiménez Hernandez, C. N. Adquisición de tecnología biomédica en IPS colombianas: comparación y mejores prácticas. Revista Gerencia y Políticas de Salud. 2016. 15(31), 88-118.

[14]. Organización Mundial de la Salud. Evaluación de Tecnologías Sanitarias Aplicada a los Dispositivos Médicos: Serie de documentos técnicos de la OMS sobre dispositivos médicos (2012). Consultado el 27 de marzo de 2019 en: http:// whqlibdoc.who.int/publications/2012/9789243501369_spa.pdf

[15]. Barrientos Gómez, J. G., Marín Castro, A. E., Becerra Ruíz, L., \& Tobón Arango, M. A. La evaluación de nuevas tecnologías en salud en hospitales: revisión narrativa. Revista Medicina UPB. 2016. 35(2). 120-134.

[16]. Duque, M., \& Lores, C. Diseño y Aplicación de un Protocolo de Evaluación de Equipos Médicos. V Latin American Congress on Biomedical Engineering CLAIB, 33. La Habana. 2013. 
[17]. Fraiz F. Organización Funcional de los Laboratorios de Análisis Clínicos. Revista de Diagnóstico Biológico. 2003. 52(1).

[18]. Usaquén Perilla, S., Cano Muñoz, A., Troncoso, D., Bonilla, N., \& de Almeida, R. El uso de la Evaluación de Tecnologías en Salud para la adquisición de tecnología en hospitales. Revista Ingeniería Biomédica. 2017. 27-34.

[19]. Kapil K. Method Development and Validation of Analytical Procedures. En: Yukihiro, S. Quality Control of Herbal Medicines and Related Areas. 2011. P. 3 - 16.

[20]. Health Information and Quality Authority. Guidelines for the Economic Evaluation of Health Technologies in Ireland: 2010.

[21]. Pacheco A, Pimentel AB, Rodríguez R, Ortiz M, Salazar R. Metodología para la Evaluación de Equipo Biomédico. Bioingeniería y Física Médica Cubana. 2002; 3(1): 22-26.

[22]. Centro Nacional de Excelencia Tecnológica en Salud (CENETEC).Evaluación de Tecnología para la Salud: Documento metodológico (2010). Consultado el 27 de marzo de 2019 en http://www.cenetec.salud.gob.mx/descargas/detes/Documento_ metodologico ETES.pdf

[23]. Osteba. Guia de Evaluación Económica en el Sector Sanitario: Departamento de Sanidad Gobierno Vasco (1999). Consultado el 27 de marzo de 2019 en: http://www.euskadi.eus/ contenidos/informacion/osteba_formacion_/en_def/adjuntos/ economiaSanitaria.pdf

[24]. Organización Mundial de la Salud. Evaluación de las necesidades de Dispositivos Médicos [Internet]. Serie de documentos técnicos de la OMS sobre dispositivos médicos (2012). Consultado el 27 de marzo de 2019 en: http://apps.who.int/medicinedocs/ documents/s21562es/s21562es.pdf

[25]. Krisrensen FB, Sugmund H. Health Technology Assessment Handbook. Copenhagen: National Board of Health. 2007.

[26]. Carvajal M, Ruiz CG. Evaluación Técnica y Clínica de Tecnología Biomédica en Procesos de Adquisición: Un enfoque en Evaluación de Tecnologías en Salud. Revista Ingeniería Biomédica. 2008; 2(4): 34 - 45. 